Irene Ciancarelli

Maria G. Tozzi-Ciancarelli

Caterina Di Massimo

Luigi Olivieri

Antonio Carolei

\section{Preventive non-pharmacological treatment and nitric oxide in chronic migraine}

Published online: 20 July 2005

I. Ciancarelli $(\bowtie) \cdot$ L. Olivieri • A. Carolei

Department of Neurology,

Regional Headache Center,

Università degli Studi di L'Aquila,

Piazzale Salvatore Tommasi 1,

I-67010 Coppito - L'Aquila, Italy

e-mail: i_ciancarelli@yahoo.it

Tel.: +39-0862-64153

Fax: +39-0862-64153

M.G. Tozzi-Ciancarelli • C. Di Massimo Laboratory of Applied Physiology,

University of L'Aquila,

I-67100 L'Aquila, Italy

\author{
Abstract In chronic migraine the \\ central sensitisation and the \\ changes of regional cerebral blood \\ flow are mediated by nitric oxide \\ (NO) and oxygen free radicals. \\ Biofeedback is considered a pre- \\ ventive non-pharmacological treat- \\ ment decreasing migraine attacks. \\ We investigated whether biofeed- \\ back effectiveness is related to \\ relaxation processes and its influ- \\ ence on oxidative stress. The \\ Migraine Disability Assessment \\ Score (MIDAS) and serum NO sta- \\ ble metabolites $\left(\mathrm{NO}_{x}\right)$ were evaluat- \\ ed in 20 patients with chronic \\ migraine before and at the end of \\ biofeedback sessions. MIDAS score
}

was lower after biofeedback than that reported before treatment. $\mathrm{NO}_{x}$ serum levels were higher after biofeedback than those measured before starting treatment. Thus, the effectiveness of biofeedback is related to a muscular relaxation and to its influence on NO bioavailability in patients with chronic migraine.

Key words Chronic migraine • Biofeedback • Nitric oxide

\section{Objectives}

The International Classification of Headache Disorders, 2nd edition, codifies Chronic migraine (1.5.1) as a new pathological entity, occurring 15 or more days per month, for more than 3 months, in the absence of medication overuse [1]. Chronic pain is related to central sensitisation and is mediated by excitatory amino acids, neurokinins and by nitric oxide (NO), involved in the regulation of the cerebral vessels tone. NO may function as a signalling molecule in controlling neuronal activity and is crucial in controlling signal-to-noise sensory inputs during migraine attack and interacting with oxygen free radicals, which may cause headache through variations of cerebral blood flow [2-4]. A preventive non-pharmacologic treatment such as biofeedback, inducing the patients to detect and interact with psychologic processes, may prove effective in decreasing the disability and improving quality of life of patients with chronic headache [5]. We investigated whether the efficacy of biofeedback might be related to relaxation processes and its influence on NO serum concentration.

\section{Methods}

Twenty patients with chronic migraine (mean age \pm SD: $25.6 \pm 4.6$ years) were examined. Chronic migraine was diagnosed according to the criteria of the Headache Classification Subcommittee 
of the International Headache Society. Headache disability were evaluated by the Migraine Disability Assessment Score (MIDAS), administered before and after the biofeedback treatment sessions. No pharmacological preventive treatment was permitted during the study. Patients were allowed to treat migraine headache if pain was so severe as to compromise daily activity. We suggested the use of indomethacin at a maximum of 50 $\mathrm{mg} /$ day. For the biofeedback session we used the Satem Myoexpand Series 400, EMG Module M3. Three circular metal electrodes were applied to the frontal muscle. Migraine sufferers underwent three sessions of biofeedback per week for a total of 12 sessions. Each biofeedback session lasted $20 \mathrm{~min}$. Serum samples collected before and after biofeedback sessions were assayed for $\mathrm{NO}$ stable metabolites $\left(\mathrm{NO}_{x}\right)$. $\mathrm{NO}_{x}$ were measured by reducing nitrate to nitrite and measuring nitrite by a sensitive fluorimetric assay based on the reaction of nitrite with 2,3-diaminonaphthalene to form the fluorescent product 1-(H)-naphthotriazole. Intensity of the fluorescent signal of the samples was measured and compared with known concentrations of sodium nitrite (excitation wavelength $375 \mathrm{~nm}$, emission wavelength $415 \mathrm{~nm}$ ).

\section{Results}

MIDAS scores of migraine attacks after biofeedbackassisted relaxation training were lower with respect to those reported before $(36.9 \pm 13.9 v s .18 .8 \pm 10.4, p<0.001)$. $\mathrm{NO}_{x}$ serum levels were higher after biofeedback training sessions than those measured before $(31.3 \pm 7.1 \mathrm{vs}$. $23.7 \pm 4.2 \mu \mathrm{M}, p<0.001)$.

\section{Discussion}

Biofeedback-assisted relaxation training is a non-invasive behavioural treatment with good compliance in migraine sufferers and without adverse events. This mind-body technique may help to treat a wide range of mental and physical health problems controlling involuntary body responses such as blood pressure, heart rate and muscle tension. The effectiveness of behavioural interventions is related to making patients aware of their bodies' physiologic responses, reducing anxiety and depression and inducing a muscular relaxation [6, 7]. After the biofeedback sessions we observed an increase in NO bioavailability. NO synthesised by the constitutive enzyme mediates mainly physiological effects, whereas the expression of inducible NO synthases may lead to a sizeable NO bioavailability associated with cytotoxic effects. The relaxation therapies may be considered useful in decreasing chronic pain activating only constitutive NO pathways. Therefore, the effectiveness of behavioural interventions such as biofeedback in limiting migraine attacks may be related to a reduction of muscular relaxation and to an increase of NO bioavailability.

\section{References}

1. Headache Classification Subcommittee of the International Headache Society (2004) The International Headache Classification of Headache Disorders, 2nd e0dn. Cephalalgia 24[Suppl $1]: 1-160$

2. Ciancarelli I, Tozzi-Ciancarelli MG, Di Massimo C et al (2003) Urinary nitric oxide metabolites and lipid peroxidation by products in migraine. Cephalalgia 23:39-42
3. Ciancarelli I, Tozzi-Ciancarelli MG, Di Massimo C et al (2004) Flunarizine effects on oxidative stress in migraine sufferers. Cephalalgia 24:528-532

4. Erdos B, Lacza Z, Toth IE et al (2003) Mechanisms of pain-induced local cerebral blood flow changes in the rat sensory cortex and thalamus. Brain Res 960(1-2):219-227

5. Andrasik F (2003) Behavioral treatment approaches to chronic headache. Neurol Sci 24[Suppl 2]:S80-S85
6. Vasudeva S, Claggett AL, Tietjen GE et al (2003) Biofeedback-assisted relaxation in migraine headache: relationship to cerebral blood flow velocity in the middle cerebral artery. Headache 43:245-250

7. Esch T, Fricchione GL, Stefano GB et al (2003) The therapeutic use of the relaxation response in stress-related diseases. Med Sci Monit 9:RA23-24 